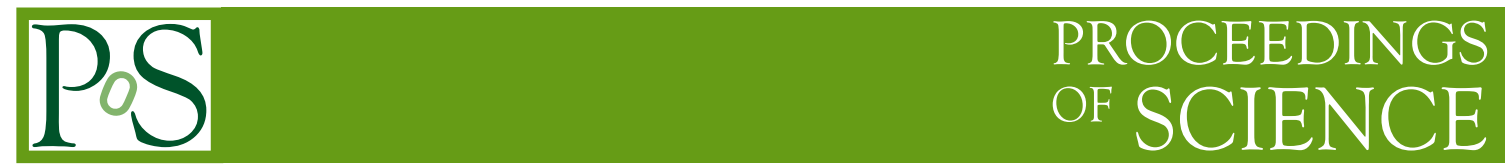

\title{
SMEFT and searches for new physics
}

\author{
Ken Mimasu ${ }^{a, *}$ \\ ${ }^{a}$ Centre for Cosmology, Particle Physics and Phenomenology (CP3), Université catholique de Louvain, \\ 1348 Louvain-la-Neuve, Belgium \\ E-mail: ken.mimasu@uclouvain.be
}

I review recent developments in searches for signs of new physics via the Standard Model Effective Field Theory framework. I focus on recent global analyses and their improvement by increased the parameter spaces, increased datasets and improved theoretical predictions. These ultimately lead to a broader and more faithful coverage of the new physics model space that can be mapped to from the data. Finally, I discuss a pathway for improving future sensitivity by pursuing high-energy and high-multiplicity final states.

The Eighth Annual Conference on Large Hadron Collider Physics-LHCP2020

25-30 May, 2020

online

${ }^{*}$ Speaker 


\section{Introduction}

During a decade-long physics programme, the LHC experiments have given us a deeper understanding of the properties and interactions of fundamental particles. Spearheaded by the Higgs boson discovery in 2012, the wealth of precision measurements and rare-process discoveries paint a picture that is consistent with the SM. Moreover, the energy frontier of the machine has been effectively saturated, with only relatively minor gains in reach, going forward. This exploration has not yet brought any clear signs of new physics at the TeV scale. One can still draw three possible scenarios from the data. The first is that new physics is very weakly coupled, and rate limited. In this case the factor $\sim 20$ of data from the HL-LHC gives hope for improvement. The second is that its signatures are too exotic, and have evaded our collective imagination. Finally, it may simply be too heavy, lying out of reach of the machine. While this is the worst case scenario for the point of view of direct searches, it opens an avenue for indirect searches.

The last possibility lends itself to the comprehensive precision measurement programme, which - thanks to the great efforts of the High Energy Physics community - is being pursued at the LHC, despite it being a hadron collider. The natural place to look for the underlying dynamics behind EW symmetry breaking is in the interactions among the key players in this mechanism, namely, the Higgs bosons, EW gauge bosons and the top quark. Independent of the outcomes of direct, new physics searches, a major aspect of the LHC legacy will constitute precision measurements of the interactions that govern this sector. On one side, the high energy of the LHC will be able to test the connection between the gauge and Higgs sectors implied by the Goldstone equivalence theorem. On the other, although the top quark is special, its interactions are relatively poorly measured and the LHC, as a top factory, has an important role to play in pinning these down.

\section{The SMEFT}

The rise of the SM effective field theory (SMEFT) in high energy physics embodies this paradigm shift from the energy to the precision frontiers. This model-independent formalism encodes modified interactions between known particles due to new states that cannot be directly produced. It consists of an operator expansion around the SM Lagrangian.

$$
\mathcal{L}_{E F T}=\mathcal{L}_{S M}+\sum \frac{c_{i}}{\Lambda^{d_{i}-4}} O_{i} .
$$

Each operator, $O_{i}$, of dimension $d_{i}$ is built from the free fields of $\mathcal{L}_{S M}$, and weighted by a WC (WC), $c_{i}$, that maps to the underlying model parameters. By measuring the $c_{i}$, we can probe new physics living at the cutoff scale, $\Lambda$, thus extending our collider reach beyond its nominal energy. Although often viewed as a parameter space for 'anomalous' interactions, the SMEFT is simply the complete theory of SM interactions respecting $S U(3)_{C} \times S U(2)_{L} \times U(1)_{Y}$ symmetry. The SM as we know it, is the subset of operators with mass dimension less than of equal to 4 . Most studies focus on the leading, non-trivial, set of operators in this expansion, of mass dimension- 6 .

\section{Improving the global picture}

The SMEFT relies on only a few, generic assumptions, offering a model-independent description of heavy new physics. Interpreting data to measure its parameters therefore requires an appropriately 
global approach; after all, we cannot know a priori which of the operators nature will generate. The ultimate goal in this effort is a global likelihood, describing the compatibility of the full SMEFT parameter space with the totality of particle physics data. Being pragmatic, however, it is reasonable to start with limited subset of parameter space and manageable datasets. This has been the focus of many studies in recent years, which include an increasing number of experimental analyses directly presenting SMEFT interpretations. The LHC Long Shutdown 2 is an opportune time to take stock on what we have learned, and SMEFT fits can serve as important benchmarking and data preservation exercises. In order to map to the largest possible set of UV models, they must be as global as possible. This means maximising both the set of WCs and input observables. In this section, I review some recent developments in this area.

In the gauge and Higgs sector, a recent study [1] combined Higgs, EW precision and Diboson data from LEP and LHC to form one of the most comprehensive global analyses to date. Flavor universality is assumed in all of the fermionic operators, including those that modify the up quark, down quark and lepton Yukawa couplings, for a total of 19 degrees of freedom. A novel aspect is the first inclusion of the Higgs Simplified Template Cross Section (STXS) measurements, designed to succeed the $\kappa$-framework by including coarse-grained differential information. Significant improvement was observed with respect to a previous analysis, with individual (marginalised) bounds reaching as high as 17(7) TeV for certain operators, as show in Fig. 1. The analysis also performs
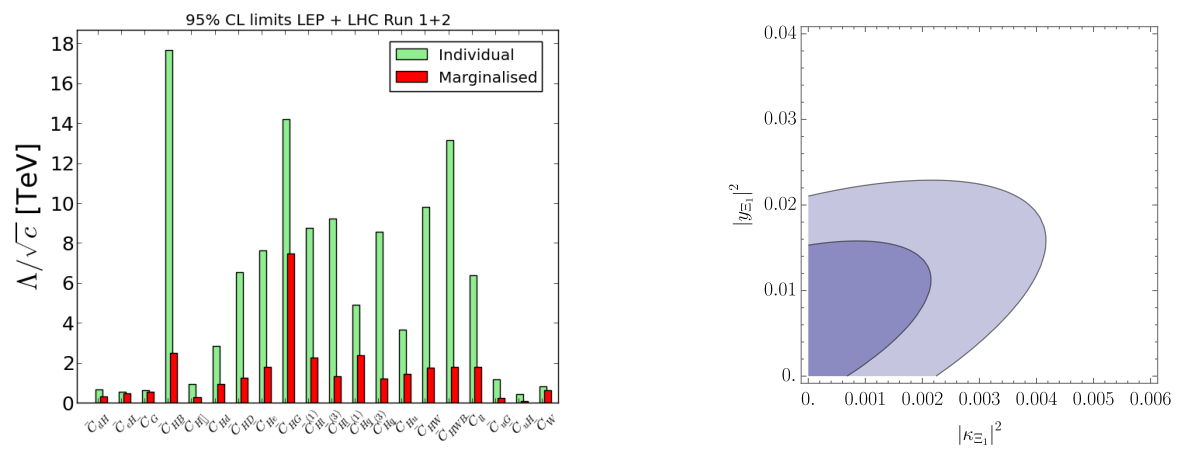

Figure 1: Left: Energy reach implied by the individual and marginalised confidence intervals on the WCs of the global fit performed in [1]. Right: Constraints on a two-parameter model describing as scalar EW triplet extension with a mass set to $1 \mathrm{TeV}$ implied by the analysis.

the important, next step of mapping the constraints onto several simple UV models. The matching procedure to the SMEFT has been extensively studied recently, yielding several important developments such as a complete tree-level dictionary for single-particle extensions of the SM [2] and the development of the Universal One-Loop Effective Action [3, 4] which greatly simplify the exercise of mapping data to real models via the SMEFT. Fig. 1 shows an example of such a mapping, to a 2-parameter model of a real EW triplet scalar with Hypercharge 1, $\Xi_{1}$, whose mass is fixed to 1 $\mathrm{TeV}$. One observes a significant sensitivity to small values of $\left|\kappa \Xi_{1}\right|$ and $\left|y_{\Xi_{1}}\right|$, which represent the coupling of $\Xi_{1}$ to a pair of Higgs and lepton fields, respectively. Ref. [5] relaxes the assumption of flavor universality in the fermion sector, allowing for generation-dependent modifications gauge and Yukawa interactions, with 31 degrees of freedom confronted with a similar dataset. Notably, the results addressed whether a relaxed flavor assumption could absorb the long-standing tension 
with the SM in the LEP $b$-quark forward-backward asymmetry, $A_{F B}^{0, b}$ [6]. Indeed, the parameters affecting the right(left)-handed $b$-quark couplings to the $Z$ exhibit pulls of 3.1(2) $\sigma$ away the SM. This allows for the other couplings to be better constrained, since the non $b$-quark observables agree much better with SM predictions. The results were also mapped to a custodial vector triplet model, probing mass scales around $4.3 \mathrm{TeV}$, assuming WCs of order 1 .

The top sector has recently seen two global fits $[7,8]$ that significantly improve new physics sensitivity. A total of 34 and 22 coefficients are fitted, respectively, including the top quark chromomagnetic moment, EW top interactions and the large set of four fermion operators. Compared to the gauge/Higgs sector, there is a lower energy reach $(0.35-2 \mathrm{TeV})$, with hierarchies in sensitivity. For example, top pair production and $W$-boson helicity fractions in top decays are two very precise measurements, while rarer processes such as single-top plus $Z$ and four-top production are poorly measured, and incorporate less differential data. The large degeneracies present in the four fermion sector have a significant degrading impact on the overall sensitivity. Finally the quadratic, $1 / \Lambda^{4}$, terms in the EFT contribution to LHC cross sections can dominate the constraining power (see Fig. 2, Right), suggesting that more data is required to ensure the validity of the EFT expansion.

Looking ahead, experimentally, the Higgs STXS programme will form an integral part of all future fits to Higgs data. The present differential data with common fiducial definitions such that they can be combined across analyses, retaining correlation information (See e.g. Refs. $[9,10]$ ). The clear next step in SMEFT interpretations is to combine Higgs/EW measurements with top data, to assess the cross-talk between the sectors, and map to a more realistic class of UV models that underpin EWSB. On the theoretical side, an important exploratory study was performed in Ref. [11] to examine the extent to which SMEFT effects could be absorbed into PDF fits using DIS data. These are typically performed in the SM hypothesis but, ideally, one should simultaneously extract the WCs. It was found that the SMEFT often provides a better fit to the DIS data and that a simultaneous fit can significantly affect the extracted limits. It is also important to connect high energy collider measurements to flavour data. Refs. $[12,13]$ examine how EW renormalisation group running effects can connect flavor-symmetric SMEFT operators to low energy measurements of flavour-changing neutral currents. It is shown that the latter measurements provide complementary constraints that close certain blind directions present in existing fits.

\section{SMEFT in loops}

The SMEFT is, like the SM, a (order-by-order in $\Lambda$ ) renormalisable quantum field theory. Hence, theoretical predictions can be systematically improved by perturbative corrections in both the SM couplings and $1 / \Lambda$. The last few years have also seen a significant push in the computations of SMEFT predictions beyond tree-level in QCD and EW gauge couplings. These improve control over uncertainties from missing higher orders, and lead to better predictions for the correlations among observables that ultimately determine our sensitivity to new physics. Moreover, a new avenue for sensitivity to operators arising at one-loop is opened. The most famous example of loop-induced sensitivity is the SMEFT contributions to Higgs production in gluon fusion via the top quark loop.

A notable development was recently presented in Ref [14], which includes, for the first time, EW loop corrections into the fit to EW precision observables. An immediate consequence of including one-loop effects is that the set of relevant operators in the Warsaw basis [15] increases 
from 10 to 32, which shows a significant increase in parameter space degeneracy considering the 8(10) constrained directions at LO(NLO). Restricting to a closed, 8-dimensional subspace of operators that can be fit to this data, shown effects of order $20-30 \%$ in the marginalised bounds (Fig. 2, Left). Along these lines, the aforementioned top-sector fits were the first to include NLO QCD corrections in the SMEFT predictions, showing $O(1)$ effects in the marginalised constraints (Fig. 2, Right). To maximise the impact of these theoretical developments, it is important that they

\begin{tabular}{|c|c|c|}
\hline Coefficient & LO & NLO \\
\hline \hline $\mathcal{C}_{\phi D}$ & {$[-0.034,0.041]$} & {$[-0.039,0.051]$} \\
\hline $\mathcal{C}_{\phi W B}$ & {$[-0.080,0.0021]$} & {$[-0.098,0.012]$} \\
\hline $\mathcal{C}_{\phi d}$ & {$[-0.81,-0.093]$} & {$[-1.07,-0.03]$} \\
\hline $\mathcal{C}_{\phi l}^{(3)}$ & {$[-0.025,0.12]$} & {$[-0.039,0.16]$} \\
\hline $\mathcal{C}_{\phi u}$ & {$[-0.12,0.37]$} & {$[-0.21,0.41]$} \\
\hline $\mathcal{C}_{\phi l}^{(1)}$ & {$[-0.0086,0.036]$} & {$[-0.0072,0.037]$} \\
\hline $\mathcal{C}_{l l}$ & {$[-0.085,0.035]$} & {$[-0.087,0.033]$} \\
\hline $\mathcal{C}_{\phi q}^{(1)}$ & {$[-0.060,0.076]$} & {$[-0.095,0.075]$} \\
\hline \hline
\end{tabular}

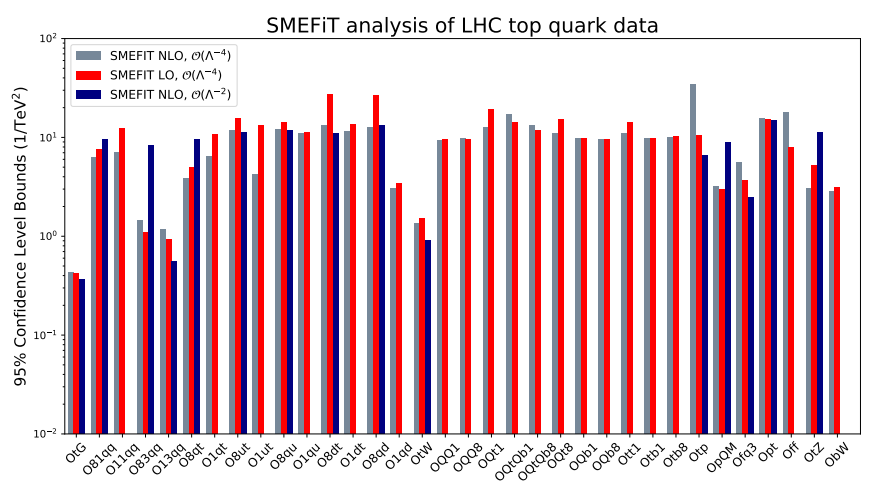

Figure 2: Comparison plots for marginalised, $95 \%$ confidence intervals on WCs in two fits. Left: Bounds at LO and NLO on an 8-parameter subspace of coefficients bounded by Z-pole measurements [14]. Right: Bounds from the top sector fit of Ref. [7] for $\Lambda=1 \mathrm{TeV}$, highlighting the impact of NLO and $1 / \Lambda^{4}$ effects.

be implemented in Monte Carlo codes. SMEFTatNLO [16] represents an important milestone in this direction, enabling automated one-loop computations for Monte Carlo event generation. A large set of WCs (including, for the first time, four fermion operators) are implemented, with a flavour assumption that singles out top quark interactions.

\section{High energy/multiplicity}

An important feature of the SMEFT is the energy growth that it predicts in scattering amplitudes with respect to the SM, thanks to its higher dimension operators. This must be exploited in full at the LHC to maximise the SMEFT sensitivity. Taking the schematic EFT dependence of a $2 \rightarrow 2$ scattering amplitude,

$$
\mathcal{A} \sim \mathcal{A}_{S M}\left(1+c_{i} \frac{v^{2}}{\Lambda^{2}}+c_{j} \frac{v E}{\Lambda^{2}}+c_{k} \frac{E^{2}}{\Lambda^{2}}\right)
$$

one can see that both energy constant and energy-growing effects are possible. The former are best constrained by precise rate measurements, and will ultimately be limited by systematic uncertainties, while the latter can be probed in increasingly differential measurements and, in principle, never reach such a limitation. The term 'energy helps accuracy' [17] describes the idea that probing SMEFT in the right processes at high energies can compensate for a lack of precision.

However, it is not guaranteed that a WC will yield energy growth in a given amplitude, and its interference with the SM amplitude may be suppressed by helicity selection [18]. These energyconstant terms of Eq. (1), are most often attributed to a Higgs field in an operator taking its vacuum expectation value. Nevertheless, there is always some amplitude in which a higher-dimensional 
operator predicts energy-growth with respect to the SM, and it is important that these are identified and exploited. One can naively understand that the presence of contact interactions in the effective Lagrangian are a likely source of this growth. For example, examining operators containing the Higgs field in Feynman gauge, bearing in mind the Goldstone equivalence theorem, allows one to identify where the amplitudes for longitudinally polarised gauge bosons will grown with energy. This has important phenomenological implications in top physics [19, 20], Higgs measurements [21] and di-boson production [22]. These contact interactions provide an important avenue to probe the SMEFT in an enriched phase space, favouring both high energy and high multiplicity.

\section{Conclusions}

The SMEFT is now the established framework for interpreting precision measurements at the LHC, an is therefore an active field of research. I have presented some recent developments in improving our global understanding of the parameter space and its implications for new physics scenarios. These come from more general and precise fits, the inclusion of one-loop effects, and the incorporation of increasingly rare, high-energy and high-multiplicity final states.

\section{References}

[1] J. Ellis, C. W. Murphy, V. Sanz, and T. You, Updated Global SMEFT Fit to Higgs, Diboson and Electroweak Data, JHEP 06 (2018) 146, [arXiv : 1803 . 03252].

[2] J. de Blas, J. Criado, M. Perez-Victoria, and J. Santiago, Effective description of general extensions of the Standard Model: the complete tree-level dictionary, JHEP 03 (2018) 109, [arXiv: 1711.10391].

[3] B. Henning, X. Lu, and H. Murayama, How to use the Standard Model effective field theory, JHEP 01 (2016) 023, [arXiv: 1412 . 1837].

[4] A. Drozd, J. Ellis, J. Quevillon, and T. You, The Universal One-Loop Effective Action, JHEP 03 (2016) 180, [arXiv: 1512.03003].

[5] A. Falkowski and D. Straub, Flavourful SMEFT likelihood for Higgs and electroweak data, JHEP 04 (2020) 066, [arXiv: 1911.07866$].$

[6] ALEPH, DELPHI, L3, OPAL, SLD, LEP Electroweak Working Group, SLD Electroweak Group, SLD Heavy Flavour Group Collaboration, S. Schael et al., Precision electroweak measurements on the $Z$ resonance, Phys. Rept. 427 (2006) 257-454, [hep-ex/0509008].

[7] N. P. Hartland, F. Maltoni, E. R. Nocera, J. Rojo, E. Slade, E. Vryonidou, and C. Zhang, $A$ Monte Carlo global analysis of the Standard Model Effective Field Theory: the top quark sector, JHEP 04 (2019) 100, [arXiv: 1901.05965 ].

[8] I. Brivio, S. Bruggisser, F. Maltoni, R. Moutafis, T. Plehn, E. Vryonidou, S. Westhoff, and C. Zhang, O new physics, where art thou? A global search in the top sector, JHEP 02 (2020) 131, [arXiv: 1910.03606]. 
[9] ATLAS Collaboration, G. Aad et al., Combined measurements of Higgs boson production and decay using up to $80 \mathrm{fb}^{-1}$ of proton-proton collision data at $\sqrt{\mathrm{s}}=13 \mathrm{TeV}$ collected with the ATLAS experiment, Phys. Rev. D 101 (2020), no. 1 012002, [arXiv: 1909. 02845].

[10] CMS Collaboration, Measurements of properties of the Higgs boson in the four-lepton final state in proton-proton collisions at $\sqrt{s}=13 \mathrm{TeV}$,

[11] S. Carrazza, C. Degrande, S. Iranipour, J. Rojo, and M. Ubiali, Can New Physics hide inside the proton?, Phys. Rev. Lett. 123 (2019), no. 13 132001, [arXiv: 1905. 05215].

[12] T. Hurth, S. Renner, and W. Shepherd, Matching for FCNC effects in the flavour-symmetric SMEFT, JHEP 06 (2019) 029, [arXiv: 1903.00500].

[13] R. Aoude, T. Hurth, S. Renner, and W. Shepherd, The impact of flavour data on global fits of the MFV SMEFT, arXiv:2003.05432.

[14] S. Dawson and P. P. Giardino, Electroweak and QCD corrections to $Z$ and $W$ pole observables in the standard model EFT, Phys. Rev. D 101 (2020), no. 1 013001, [arXiv: 1909.02000].

[15] B. Grzadkowski, M. Iskrzynski, M. Misiak, and J. Rosiek, Dimension-Six Terms in the Standard Model Lagrangian, JHEP 10 (2010) 085, [arXiv : 1008 . 4884].

[16] C. Degrande, G. Durieux, F. Maltoni, K. Mimasu, E. Vryonidou, and C. Zhang, Automated one-loop computations in the SMEFT, arXiv:2008.11743.

[17] M. Farina, G. Panico, D. Pappadopulo, J. T. Ruderman, R. Torre, and A. Wulzer, Energy helps accuracy: electroweak precision tests at hadron colliders, Phys. Lett. B 772 (2017) 210-215, [arXiv: 1609.08157].

[18] A. Azatov, R. Contino, C. S. Machado, and F. Riva, Helicity selection rules and noninterference for BSM amplitudes, Phys. Rev. D 95 (2017), no. 6065014 , [arXiv: 1607.05236].

[19] J. A. Dror, M. Farina, E. Salvioni, and J. Serra, Strong $t W$ Scattering at the LHC, JHEP 01 (2016) 071, [arXiv: 1511.03674].

[20] F. Maltoni, L. Mantani, and K. Mimasu, Top-quark electroweak interactions at high energy, JHEP 10 (2019) 004, [arXiv: 1904 .05637].

[21] B. Henning, D. Lombardo, M. Riembau, and F. Riva, Measuring Higgs Couplings without Higgs Bosons, Phys. Rev. Lett. 123 (2019), no. 18 181801, [arXiv: 1812. 09299].

[22] Z. Zhang, Time to Go Beyond Triple-Gauge-Boson-Coupling Interpretation of W Pair Production, Phys. Rev. Lett. 118 (2017), no. 1 011803, [arXiv: 1610. 01618]. 